\title{
Cell therapy with human $T$ regulatory type 1 cells in allogeneic transplantations
}

\author{
Rosa Bacchetta ${ }^{+1}$, Silvia \\ Gregori' ${ }^{1}$, Barbarella \\ Lucarelli² \& Maria \\ Grazia Roncarolo ${ }^{1,3}$ \\ ${ }^{1}$ San Raffaele Telethon Institute for \\ Gene Therapy (HSR-TIGET), \\ Department of Regenerative \\ Medicine, Stem Cells and Gene \\ Therapy, Milan 20132, Italy \\ ${ }^{2}$ Department of Paediatric \\ Haematology-Oncology, IRCCS, \\ Ospedale Bambino Gesu, Rome, Italy \\ ${ }^{3}$ Vita Salute San Raffaele University, \\ Milan, Italy \\ ${ }^{+}$Author for correspondence: \\ rosa.bacchetta@hsr.it
}

\section{Financial \& competing}

\section{interests disclosure}

The authors have no relevant affiliations or financial involvement with any organization or entity with a financial interest in or financial conflict with the subject matter or materials discussed in the manuscript. This includes employment, consultancies, honoraria, stock ownership or options, expert testimony, grants or patents received or pending, or royalties.

No writing assistance was utilized in the production of this manuscript.
Regulatory T cells (Tregs) are a specialized subset of T cells able to control immune responses and promote and maintain immune tolerance. Deficiency or defective function of Tregs has been correlated with autoimmunity, whereas their presence has been associated with tolerance. $\mathrm{CD}^{+}$Tregs have been classified into two major subsets according to their origin: the natural occurring Tregs, which develop in the thymus and are present in mice and healthy human individuals since birth, and the inducible Tregs that are generated in the periphery under different tolerogenic conditions. Among the inducible Tregs, the T regulatory type 1 (Tr1) cells represent one of the most extensively characterized subset. Tr1 cells develop in the periphery upon chronic antigen stimulation in the presence of IL-10 produced by tolerogenic antigen-presenting cells. Tr1 cells are characterized by the capacity to produce high levels of IL-10 in the absence of IL-4 and can suppress undesired immune responses mainly through cytokine-mediated mechanisms. However, we recently demonstrated that immunomodulatory activities of Tr1 cells reside not only in their ability to secrete suppressive cytokines but also in their property of cell-to-cell contact-dependent killing of target myeloid cells mediated by granzyme B and perforin. Tr1 cells are distinct from natural occurring Tregs since they are independent from FOXP3 expression for both their function and generation, as demonstrated by Tr1 cell identification in IPEX patients. The presence of $\operatorname{Tr} 1$ cells in vivo is associated with tolerance, whereas defects in $\operatorname{Tr} 1$ cells lead to autoimmune diseases or to chronic inflammation. In humans, we showed that both exogenous IL-10 or IL-10-derived from tolerogenic dendritic cells (DC-10) can be used to generate alloantigen-specific Tr1 cells in vitro suitable for cell therapy. Moreover, a homogeneous population of IL-10-producing T cells with Tr1-like phenotype and functions can be generated by transducing human $\mathrm{CD}^{+}{ }^{+} \mathrm{T}$ cells with a lentiviral vector encoding for human IL-10. A cellular therapy protocol for the ex vivo use of IL-10 to induce alloantigen-specific unresponsiveness in donor-derived T cells for adoptive transfer in patients transplanted with haploidentical hematopoietic stem cells is being applied for patients with highrisk hematopoietic malignancies. In our clinical trial, donor T cells anergized in vitro in the presence of IL-10 are infused post-transplantation into the host with the ultimate goal of providing immune reconstitution with donor T cells that are anergic towards host antigens and contain precursors of host-specific Tr1 cells. This cellular therapy has proven to be safe and to provide immunoreconstitution associated, in most of the patients, with only moderate graft-versus-host disease (GVHD) at a cell dose that, if transferred untreated, would have caused severe acute GVHD. A similar approach could be also foreseen in the clinical setting of HLA-matched unrelated donor to prevent acute and chronic GVHD. Ad hoc experimental settings have been established using dendritic cells as antigen-presenting cells to induce anergy in the context of HLA-matched donors. These recently developed methods allowing the ex vivo induction of Tregs for in vivo infusion represent the first step toward an enlarged use of these cells as cellular therapy not only to inhibit GVHD after allogeneic hematopoietic stem cell transplantation, but also to other transplantation settings, or to re-establish tolerance in autoimmune or allergic diseases.

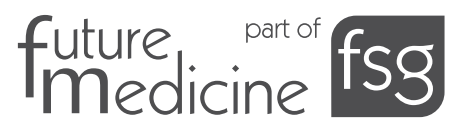

